\title{
Hybrid Hydrogels for Synergistic Periodontal Antibacterial Treatment with Sustained Drug Release and NIR-Responsive Photothermal Effect
}

This article was published in the following Dove Press journal: International Journal of Nanomedicine

\author{
Jian $\operatorname{Lin}^{1, *}$ \\ Zhiqi $\mathrm{He}^{2, *}$ \\ Fen $\mathrm{Liu}^{3, *}$ \\ Jie Feng ${ }^{4}$ \\ Chengyi Huang 5 \\ Xueli Sun ${ }^{4}$ \\ Hui Deng' \\ 'Department of Periodontics, School \& \\ Hospital of Stomatology, Wenzhou \\ Medical University, Wenzhou, Zhejiang, \\ People's Republic of China; ${ }^{2}$ Department \\ of Pediatric Dentistry, School \& Hospital \\ of Stomatology, Wenzhou Medical \\ University, Wenzhou, Zhejiang, People's \\ Republic of China; ${ }^{3}$ Department of \\ Histology and Embryology, Wenzhou \\ Medical University, Wenzhou, Zhejiang, \\ People's Republic of China; ${ }^{4}$ Engineering \\ Research Center of Clinical Functional \\ Materials and Diagnosis \& Treatment \\ Devices of Zhejiang Province, Wenzhou \\ Institute, University of Chinese Academy \\ of Sciences, Wenzhou, Zhejiang, People's \\ Republic of China; ${ }^{5}$ Department of \\ Dentistry, Sir Run Run Shaw Hospital, \\ School of Medicine, Zhejiang University, \\ Hangzhou, Zhejiang, People's Republic of \\ China
}

*These authors contributed equally to this work
Background: Periodontal pathogenic bacteria promote the destruction of periodontal tissues and cause loosening and loss of teeth in adults. However, complete removal of periodontal pathogenic bacteria, at both the bottom of the periodontal pocket and the root bifurcation area, remains challenging. In this work, we explored a synergistic antibiotic and photothermal treatment, which is considered an alternative strategy for highly efficient periodontal antibacterial therapy.

Methods: Mesoporous silica (MSNs) on the surface of Au nanobipyramids (Au NBPs) were designed to achieve the sustained release of the drug and photothermal antibacterials. The mesoporous silica-coated Au NBPs (Au NBPs@SiO $\mathrm{Si}_{2}$ ) were mixed with gelatin methacrylate (GelMA-Au NBPs@SiO 2 ). Au NBPs@SiO 2 and GelMA-Au NBPs@SiO $\mathrm{S}_{2}$ hybrid hydrogels were characterized, and the drug content and photothermal properties in terms of the release profile, bacterial inhibition, and cell growth were investigated.

Results: The GelMA-Au NBPs@ $\mathrm{SiO}_{2}$ hybrid hydrogels showed controllable minocycline delivery, and the drug release rates increased under $808 \mathrm{~nm}$ near-infrared (NIR) light irradiation. The hydrogels also exhibited excellent antibacterial properties, and the antibacterial efficacy of the antibiotic and photothermal treatment was as high as $90 \%$ and $66.7 \%$ against Porphyromonas gingivalis ( $P$. gingivalis), respectively. Moreover, regardless of NIR irradiation, cell viability was over $80 \%$ and the concentration of $\mathrm{Au} \mathrm{NBPs} @ \mathrm{SiO}_{2}$ in the hybrid hydrogels was as high as $100 \mu \mathrm{g} / \mathrm{mL}$.

Conclusion: We designed a new near-infrared light (NIR)-activated hybrid hydrogel that offers both sustained release of antibacterial drugs and photothermal treatment. Such sustained release pattern yields the potential to rapidly eliminate periodontal pathogens in the periodontal pocket, and the photothermal treatment maintains low bacterial retention after the drug treatment.

Keywords: periodontitis, mesoporous silica, $\mathrm{Au}$ nanobipyramids, gelatin methacrylate, photothermal therapy, antibacterial properties

\section{Introduction}

Periodontitis, which is one of the most common chronic oral infectious diseases, is initiated by periodontopathic bacteria, resulting in the formation of periodontal pocket and the destruction of the surrounding and supporting tissues of the teeth. ${ }^{1-3}$ Typically, mechanical debridement by scaling and root planing (SRP) to remove the subgingival pathogens is conventionally performed in non-surgical periodontal therapy. But it does not completely remove subgingival pathogens, especially in deep pockets, owing to the complex tooth anatomy. ${ }^{4,5}$ Therefore, several antibiotics are used to clear the residual bacteria. ${ }^{6}$ However, the destruction of normal flora and difficulty in maintaining local
Correspondence: Hui Deng; Xueli Sun Email dh0726@163.com;

Sunxl@wibe.ac.cn
International Journal of Nanomedicine 2020:I5 5377-5387

5377 
therapeutic concentrations have limited the use of antibiotics. $^{7}$ And overuse of antibiotics has led to the emergence of the drug-resistant bacteria, thus making antibiotic treatments ineffective. Therefore, it is vital to develop a new strategy for not only achieving the removal of residual bacteria in the early stage of treatment, but also for maintaining a low number of bacteria in the long term.

In previous decades, Mesoporous silica (MSNs) have been received much attention as drug carriers, including for the delivery of antimicrobials to treat infectious diseases. Because of the ordered pores and highly specific surface area, MSNs can carry large antibiotic loads and achieve sustained release. ${ }^{8,9}$ Further, nanovalves and polymers are applied on the surface of MSNs to protect the antibiotics loaded in the pores and target the lesion this structure on the surface can be activated by $\mathrm{pH}^{10}{ }^{10}$ light, ${ }^{11}$ radical $^{12}$ and enzyme. ${ }^{13}$ However, when the concentration of antibiotics loaded onto the MSNs decreases to below the lowest effective concentration, subgingival pathogens resume proliferation, leading to recurrence of infection.

Photothermal is widely applied in medical treatment due to its spatiotemporally controlled photothermal effect by photo-induced heat. ${ }^{14}$ Particularly, photothermal therapy (PTT) induced by near-infrared (NIR) can effectively kill bacteria with deep tissue penetration and little photodamage, making it a good supplement to conventional medical treatment. ${ }^{15,16}$ Among numerous photothermal nanomaterials, Au nanobipyramids (Au NBPs) have been emerged as an excellent photothermal agent because of the strong surface plasmon resonance, good biocompatibility and efficient PTT conversion performance. ${ }^{17}$ Therefore, Au NBPs can be used as an excellent photothermal reagent with NIR controllability for synergistic phototherapy and pharmacotherapy.

Here, we designed a hybrid hydrogel containing highsurface area MSNs and Au NBPs with photothermal properties. The large surface area of Au NBPs@ $\mathrm{SiO}_{2}$ enables the MSNs to load and release the antibiotics, and also achieves long-term control of bacterial proliferation on the tooth surface. Additionally, the incorporation of Au NBPs@ $@ \mathrm{SiO}_{2}$ into the GelMA hydrogel greatly enhanced biocompatibility with the periodontal microenvironment and increased the duration of retention in the periodontal pocket.

\section{Materials and Methods}

\section{Materials}

Gold chloride trihydrate $\left(\mathrm{HAuCl}_{4} \cdot 3 \mathrm{H}_{2} \mathrm{O}, 48 \%\right)$, sodium borohydride $\left(\mathrm{NaBH}_{4}, 98 \%\right)$, silver nitrate $\left(\mathrm{AgNO}_{3}, 99 \%\right)$,
L-ascorbic acid (99\%), hexadecyltrimethylammonium bromide (CTAB, 98\%), cetyltrimethylammonium (CTAC solution $25 \%$ in water), trisodium citrate (99\%), ammonia solution, minocycline (MINO), and Ingacure 2959 were obtained from Sigma-Aldrich. Hydrogen peroxide solution (30 $\mathrm{wt} \%$ ), tetraethyloxysilane (TEOS), and GelMA were purchased from Aladdin Reagent Co. Ltd. Sodium hydroxide $(\mathrm{NaOH})$, and methanol $\left(\mathrm{CH}_{3} \mathrm{OH}\right)$ were purchased from Sinopharm Chemical Reagent Limited Corporation and used as received. Trypsin-EDTA $(0.25 \%)$, fetal bovine serum (FBS), Dulbecco's modified Eagle medium (DMEM), Hank's Balanced Salt Solution, LIVE/DEAD ${ }^{\mathrm{TM}}$ Viability/Cytotoxicity Kit, LIVE/DEAD ${ }^{\mathrm{TM}}$ BacLight $^{\mathrm{TM}}$ Bacterial Viability Kit and 5-diphenyl-2-H-tetrazolium bromide (MTT) were purchased from Life Technologies. Brain Heart Infusion (BHI), hemin and vitamin $\mathrm{K}$ were purchased from the Solarbio. Water was purified with a Millipore system, and all glassware and Teflon-coated magnetic stirring bars were thoroughly cleaned with aqua regia, followed by copious rinsing with purified water.

\section{Synthesis of Au NBPs and Au $\mathrm{NBPs@SiO} 2$}

The Au NBPs were synthesized by a seed-mediated method, according to a previous report. ${ }^{18}$ Firstly, $0.15 \mathrm{~mL}$ of $10 \mathrm{mM}$ $\mathrm{NaBH}_{4}$ solution was added to an aqueous solution composed of $0.125 \mathrm{~mL}$ of $10 \mathrm{mM} \mathrm{HAuCl}_{4}, 0.25 \mathrm{~mL}$ of $10 \mathrm{mM}$ trisodium citrate, and $9.625 \mathrm{~mL}$ of water. A seed solution was obtained in a water bath maintained at $30{ }^{\circ} \mathrm{C}$ for 2 h. Secondly, $20 \mathrm{~mL}$ of $10 \mathrm{mM} \mathrm{HAuCl}_{4}$ was mixed with $400 \mathrm{~mL}$ of $100 \mathrm{mM}$ CTAB solution to prepare a growth solution. Briefly, $4.0 \mathrm{~mL}$ of $10 \mathrm{mM}$ silver nitrate, $8.0 \mathrm{~mL}$ of 1.0 M hydrochloric acid, and $3.2 \mathrm{~mL}$ of $100 \mathrm{mM}$ L-ascorbic acid were added into the solution to prepare a growth solution. Then, $2.74 \mathrm{~mL}$ of the seed solution was added to the above growth solution and kept at $27{ }^{\circ} \mathrm{C}$ for several hours. A $450 \mathrm{~mL}$ solution of as-synthesized Au NBPs was centrifuged at $7500 \mathrm{rpm}$ for $15 \mathrm{mins}$; then, the precipitates were redispersed in $315 \mathrm{~mL}$ of $80 \mathrm{mM} \mathrm{CTAC}$ solution and mixed with $52 \mathrm{~mL}$ of $10 \mathrm{mM} \mathrm{AgNO}_{3}$ and $26 \mathrm{~mL}$ of $100 \mathrm{mM}$ ascorbic acid. The resultant solution was kept in the water bath at $65^{\circ} \mathrm{C}$ for $4 \mathrm{~h}$ and subsequently centrifuged to enable precipitation. The precipitates were dispersed in $315 \mathrm{~mL}$ of $50 \mathrm{mM}$ CTAB and left undisturbed at $27{ }^{\circ} \mathrm{C}$ for $4 \mathrm{~h}$. The particles precipitated to the bottom of the container. The remaining precipitate was dispersed in $200 \mathrm{~mL}$ of $50 \mathrm{mM}$ CTAB solution. The resulting solution was mixed with 
$4.2 \mathrm{~mL}$ of $\mathrm{NH}_{3} \cdot \mathrm{H}_{2} \mathrm{O}(25 \mathrm{wt} \%)$ and $3.2 \mathrm{~mL}$ of $1.6 \mathrm{M} \mathrm{H}_{2} \mathrm{O}_{2}$ and kept undisturbed for $6 \mathrm{~h}$. Then, the clear supernatant was removed and centrifuged at $7500 \mathrm{rpm}$ for 15 mins. Finally, the purified Au NBPs were dispersed in $100 \mathrm{~mL}$ of $2 \mathrm{mM}$ CTAB solution and stored at $4{ }^{\circ} \mathrm{C}$ for further use.

The mesoporous silica coating Au NBPs were generated by a modified Stober method. ${ }^{19}$ Firstly, $1 \mathrm{~mL}$ of 100 $\mathrm{mM} \mathrm{NaOH}$ solution was added to $100 \mathrm{~mL} \mathrm{Au} \mathrm{NBPs,}$ adjusting the $\mathrm{pH}$ value to 11 . Then, $2.4 \mathrm{~mL}$ of $20 \%$ TEOS in methanol solution was added to the resultant solution at the rate of $0.8 \mathrm{~mL}$ every 30 mins. The mixture could react at $27{ }^{\circ} \mathrm{C}$ for $24 \mathrm{~h}$. The resulting $\mathrm{Au}$

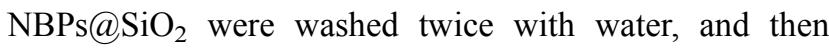
three times with methanol, and finally dispersed into $100 \mathrm{~mL}$ of methanol. The removal of CTAB from the assynthesized solution was carried out by $\mathrm{NH}_{4} \mathrm{NO}_{3}-\mathrm{CH}_{3} \mathrm{OH}$ extraction. Firstly, $5 \mathrm{~mL}$ of $\mathrm{Au} \mathrm{NBPs} @ \mathrm{SiO}_{2}$ methanol solution was added to $30 \mathrm{mg}$ of $\mathrm{NH}_{4} \mathrm{NO}_{3}$. Then, the resultant mixture was heated to reflux at $65{ }^{\circ} \mathrm{C}$ with stirring. The extracted Au NBPs@ $@ \mathrm{SiO}_{2}$ was washed with methanol for three times, and stored at $4{ }^{\circ} \mathrm{C}$.

\section{Synthesis of GelMA-Au NBPs@SiO}

Ingacure $2959(0.05 \mathrm{~g})$ was dissolved in a $10 \mathrm{~mL}$ of deionized water to yield a $0.05 \%(\mathrm{w} / \mathrm{v})$ Ingacure 2959 solution. GelMA ( $5 \mathrm{~g}$ ) was dissolved in $5 \mathrm{~mL}$ of the Ingacure 2959 solution to obtain a $10 \%$ (w/v) GelMA solution, which was fully dissolved by magnetic stirring at $60{ }^{\circ} \mathrm{C}$ for 10 mins. $100 \mu \mathrm{g} / \mathrm{mL}$ of Au NBPs@ $\mathrm{SiO}_{2}$ was centrifuged and dispersed in a GelMA solution to prepare a GelMA-Au NBPs@ $\mathrm{SiO}_{2}$ solution. This solution was added to a PMMA mold to prepare disc-shaped GelMA$\mathrm{Au} \mathrm{NBPs@SiO} 2$ under UV.

\section{Characterization}

The morphology characterizations were carried out with both scanning electron microscopy (SEM) and transmission electron microscope (TEM). SEM images were collected by using a Hitachi (SU8010) instrument. Both Au NBPs and Au $\mathrm{NBPs@SiO}{ }_{2}$ were placed on silica wafers and dried at room temperature. The samples were coated with gold, the spray time lasts 30 seconds. Moreover, TEM images $\mathrm{Au}$ NBPs@ $\mathrm{SiO}_{2}$ were collected by using a FEI Talos 200s instrument, with an operation voltage of $200 \mathrm{kV}$. The typical $\mathrm{UV}$-vis absorbance spectra of both $\mathrm{Au}$ NBPs and $\mathrm{Au}$ NBPs@ $\mathrm{SiO}_{2}$ were determined by UV-vis spectrometer (Lambda 25, PerkinElmer, USA) at a wavelength range between 450 and $900 \mathrm{~nm}$. Fourier transform infrared (FT-
IR, Tensor II, BRUKER, Germany) spectroscopy was employed to identify the surface functional structures of $\mathrm{Au}$ NBPs@SiO $\mathrm{Si}_{2}$. The collecting range was set at $500-4000 \mathrm{~cm}^{-1}$ with a resolution of $1 \mathrm{~cm}^{-1}$. The size distribution and zeta potential of Au NBPs and Au NBPs@ $@ \mathrm{SiO}_{2}$ were measured by dynamic light scattering and zeta potential measurement (ZS ZEN3600, Malvern, UK). The drug loading capacity of

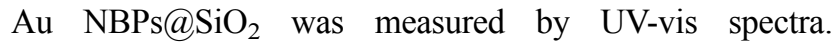
Moreover, SEM was also adopted to investigate the morphology of GelMA-Au NBPs@SiO $\mathrm{SiO}_{2}$. Briefly, GelMA-Au $\mathrm{NBPs} @ \mathrm{SiO}_{2}$ was snap frozen at $-80{ }^{\circ} \mathrm{C}$ and lyophilized for $24 \mathrm{~h}$ before coated with ultrathin gold. The images were taken at $5.0 \mathrm{kV}$. The mechanical properties of GelMA-Au $\mathrm{NBPs@SiO}{ }_{2}$ were characterized using a rheometer (DHR-2, TA, USA). The energy storage and loss modulus distribution of the GelMA samples were obtained at a shear strain of $0.5 \%$ and a frequency range of 0.1 to $10 \mathrm{~Hz}$.

\section{Photothermal Effects Under 808 nm Laser Irradiation}

The photothermal conversion efficiency of GelMA-Au $\mathrm{NBPs} @ \mathrm{SiO}_{2}$ was measured using the following method. The NIR laser light $(808 \mathrm{~nm})$ with the power of $0,0.4,0.8$ and $1.2 \mathrm{~W} / \mathrm{cm}^{2}$ was focused to a spot size of $1 \mathrm{~cm}$. The GelMA hydrogels containing 0, 25, 50, 75, and $100 \mu \mathrm{g} / \mathrm{mL}$ $\mathrm{Au} \mathrm{NBPs} @ \mathrm{SiO}_{2}$ swelled in the phosphate-buffered saline (PBS) to reach equilibrium under light irradiation, and the temperature was recorded at $30 \mathrm{~s}$ intervals for a total of 5 mins using the Thermal Imager (FLIR, E40, with an accuracy of $0.1{ }^{\circ} \mathrm{C}$ ).

\section{Release of Minocycline in vitro}

To determine the release trend of minocycline (MINO) from the GelMA hydrogels, the samples were immersed in PBS $(\mathrm{pH}=7.4)$ at $37^{\circ} \mathrm{C}$. The dried samples were immersed in $10 \mathrm{~mL}$ of PBS. At time intervals of $0.25,0.5,1,1.5,2,3,5$ and $6 \mathrm{~d}, 300 \mu \mathrm{L}$ of the solution was replaced with fresh PBS and the amount of released MINO was determined by UV-vis. In order to determine the effect of NIR light on MINO release, the samples were monitored under different conditions (with $0,0.4,0.8,1.2 \mathrm{~W} / \mathrm{cm}^{2}, 808 \mathrm{~nm}$ NIR light). At time intervals of $10,20,30,50$ and 60 mins, the amount of released MINO was determined by UV-vis. To determine the effect of collagenase on MINO release, the samples were immersed in $10 \mathrm{~mL}$ of $0.05(\mathrm{v} / \mathrm{w}) \%$ collagenase (125 CDU mg ${ }^{-1}$, solid) in Hank's Balanced Salt Solution at $37^{\circ} \mathrm{C}$. At time intervals of $0.25,0.5$, $1,1.5,2,3,5$ and $6 \mathrm{~d}, 300 \mu \mathrm{L}$ of the solution was replaced with 
fresh collagenase solution and the amount of released MINO was determined by UV-vis spectra.

\section{The Culture of Porphyromonas gingivalis and L929 Cell}

Porphyromonas gingivalis (P. gingivalis) (ATCC 33,277) was grown in Brain Heart Infusion (BHI) and BHI blood agar plates, supplemented with hemin $(5 \mu \mathrm{g} / \mathrm{mL})$ and vita$\min \mathrm{K}(1 \mu \mathrm{g} / \mathrm{mL})$ in an anaerobic chamber $\left(37^{\circ} \mathrm{C}, \mathrm{N}_{2}: \mathrm{H}_{2}\right.$ : $\left.\mathrm{CO}_{2} / 75: 10: 15\right)$.

The $\mathrm{L} 929$ cell line was maintained in DMEM supplemented with $20 \%$ FBS, penicillin (100 units $/ \mathrm{mL})$ and streptomycin $(100 \mu \mathrm{g} / \mathrm{mL})$.

\section{In vitro Antibacterial Effects}

The in vitro antibacterial effects of MINO-GelMA-Au

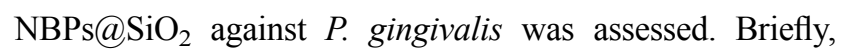
$200 \mu \mathrm{L}$ of bacterial suspension $\left(10^{7} \mathrm{CFU} / \mathrm{mL}\right)$ was incubated with GelMA-Au NBPs@SiO $\mathrm{SiO}_{2}$ and MINO-GelMA-Au NBPs@ $\mathrm{SiO}_{2}$ for 1, 3 and $5 \mathrm{~d}$ in $\mathrm{BHI}$ medium. P. gingivalis culture without nanoparticles was served as control. The growth of $P$. gingivalis was subsequently determined by measuring the optical density at a wavelength of $660 \mathrm{~nm}$ $\left(\mathrm{OD}_{600 \mathrm{~nm}}\right)$ with a SpectraMax M2 microplate reader (Molecular Devices, USA). After removing the supernatants, the bacterial were washed twice wish PBS before staining with LIVE/DEAD ${ }^{\mathrm{TM}}$ BacLight $^{\mathrm{TM}}$ Bacterial Viability Kit. The bacterial vitality was visualized by ZEISS Axio Vert. A1 fluorescence microscopy imaging system.

To further evaluate the effects of the photothermal treatment on bacteria, $200 \mu \mathrm{L}$ of bacterial suspension $\left(10^{6} \mathrm{CFU} / \mathrm{mL}\right)$ cultured in BHI medium was added to 96well plates with or without GelMA-Au NBPs@SiO $\mathrm{Si}_{2}$ and MINO-GelMA-Au NBPs@SiO . Subsequently, 0, 0.4, 0.8, and $1.2 \mathrm{~W} / \mathrm{cm}^{2} \mathrm{NIR}$ was used to irradiate the samples for 1,3 , and 5 mins. After $24 \mathrm{~h}$ of incubation, $P$. gingivalis growth was determined by $\mathrm{OD}_{600 \mathrm{~nm}}$ and $P$. gingivalis vitality was visualized by live/dead assay as described above. In addition, bacterial suspensions were serially diluted in PBS and plated on blood agar plates for the quantitation of live bacterial cells by counting CFU (CFU/mL) at day 5 .

\section{In vitro Cytotoxicity Studies}

The in vitro cytotoxicity of GelMA-Au NBPs@ $@ \mathrm{SiO}_{2}$ against L929 cells was determined by the 3- [4, 5-dimethylthiazol2-yl]-2,5-diphenyl tetrazolium bromide (MTT) and live/dead assay. Briefly, L929 cells were cultured in DMEM with different concentrations of $\mathrm{Au} \mathrm{NBPs} @ \mathrm{SiO}_{2}$ embedded in GelMA at $37{ }^{\circ} \mathrm{C}$ for $24 \mathrm{~h}$. Thereafter, $20 \mu \mathrm{L}$ of MTT solution was added and incubated for another $4 \mathrm{~h}$. The supernatant was dissolved in $150 \mu \mathrm{L}$ of DMSO and $\mathrm{OD}_{570 \mathrm{~nm}}$ was measured by SpectraMax M2 microplate reader (Molecular Devices, USA). Moreover, LIVE/DEAD ${ }^{\mathrm{TM}}$ Viability/Cytotoxicity Kit was used to detect cell vitality after the indicated treatment. L929 were stained with LIVE/DEAD ${ }^{\text {TM }}$ Viability/Cytotoxicity Kit and observed using a ZEISS Axio Vert.A1 fluorescence microscopy imaging system and count the number of cells by ImageJ software.

In order to evaluate the cytotoxicity in the presence of NIR light, cells were seeded onto the hydrogels, followed by $0.4,0.8$ and $1.2 \mathrm{~W} / \mathrm{cm}^{2}$ NIR light irradiation for 1,3 and 5 mins, respectively. After culturing for $24 \mathrm{~h}$, the in vitro cytotoxicity of the hydrogels was determined by MTT and live/dead assay as described above.

\section{Statistical Analysis}

All assays were performed in triplicate, and each treatment tested was repeated three times. All values are presented as mean $\pm \mathrm{SD}$, and calculated by one-way ANOVA with the LSD test for multiple comparisons. All statistical analyses were executed with the SPSS 19.0 software, with $\mathrm{p}<0.05$ were regarded as statistically significant.

\section{Results and Discussion Characterization of Au NBPs@SiO}

The synthesis of the Au NBPs@ $\mathrm{SiO}_{2}$ core@shell nanostructure involves seed-mediated growth and modified Stober methods. ${ }^{19}$ As shown in Figure 1A, representative SEM images of Au NBPs indicate that the average length and width of Au NBPs are about 107 and $32 \mathrm{~nm}$, respectively. After coating mesoporous silica, the Au NBPs@ $\mathrm{SiO}_{2}$ show the typical core-shell structure, with an average shell thickness of $11 \mathrm{~nm}$ (as shown in Figure 1B and C). The corresponding UVvis spectra further confirms the silica layers of Au NBPs (Figure 1D) based on the shift of the peak of longitudinal surface plasmon resonance (LSPR) from 808 to $818 \mathrm{~nm}$ after application of the silica shell coating. According to the MieGans theory, the increasing dielectric via the silica coating enhances the plasmon wavelength and leads to the red shift. ${ }^{20}$ This result is consistent with previous report, in which the local refractive index of the surrounding medium for Au NBPs lead to the red shift of LSPR peak after replacing CTAB ligands with silica shell. ${ }^{21}$ The FT-IR spectra were generated to evaluate the chemical structure of Au NBPs@SiO 2 (Figure 1E). 

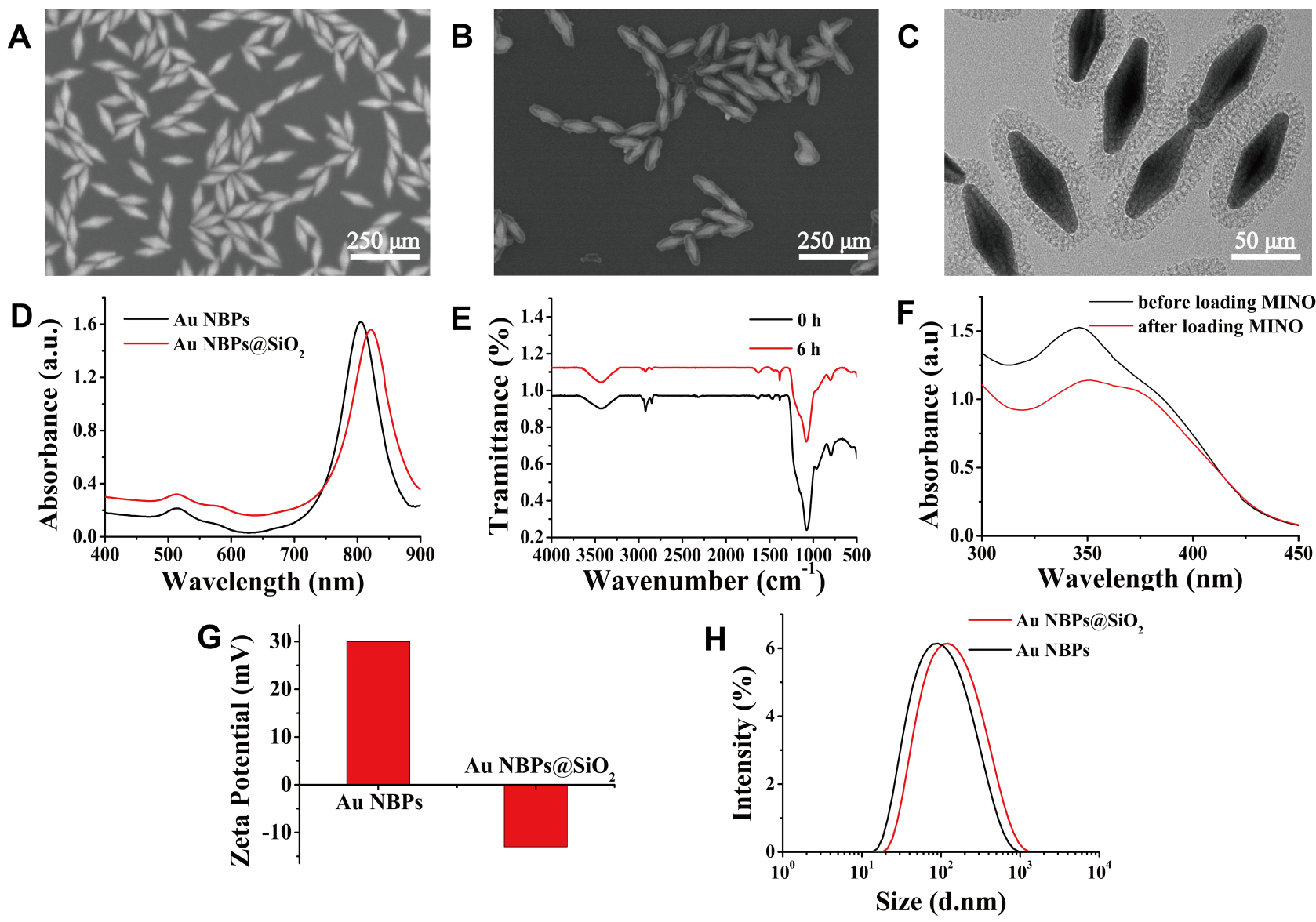

Figure I The characterization of Au NBPs@SiO 2 . SEM image of Au NBPs (A) before and (B) after silica coating. Scale bar = $250 \mu$ m. (C) TEM image of Au NBPs@SiO 2 .

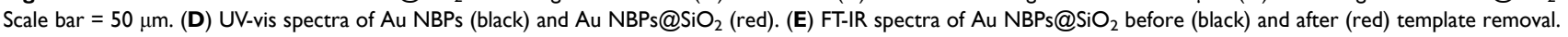
(F) UV-vis spectra of the MINO solution before being loaded onto Au NBPs@SiO 2 (black) and after being loaded onto the Au NBPs@SiO 2 (red). (G) The zeta potential of Au NBPs and Au NBPs@SiO . (H) The size distribution of Au NBPs (black) and Au NBPs@SiO 2 (red).

A broad peak from $3600 \mathrm{~cm}^{-1}$ to $3300 \mathrm{~cm}^{-1}$ is attributed to the hydroxyl groups from the silica surface. Additionally, three typical chemical wavenumbers belonging to Si-O bonds are located at $1080 \mathrm{~cm}^{-1}$. The removal of CTAB is also confirmed by FT-IR spectra. Both the intensities of asymmetrical and symmetrical vibrations at 2930 and $2850 \mathrm{~cm}^{-1}$ from $\mathrm{C}-\mathrm{H}$ bond from CTAB reduce sharply. As mentioned above, the electrostatic force between the oligomeric silicate anions and cationic headgroups $(\mathrm{CTA}+)$ of the $\mathrm{CTAB}$ is broken by the introduction of $\mathrm{NH}_{4} \mathrm{NO}_{3}$ in methanol. ${ }^{19}$ And it indicates that most of CTAB has been successfully extracted and get a more open and hollower pore channel to increase its drug loading capacity. The successful loading of MINO is detected by UVvis spectra (Figure 1F). The typical absorption of $\mathrm{Au}$ $\mathrm{NBPs} @ \mathrm{SiO}_{2}$ occurs at $345 \mathrm{~nm}$. After loading MINO, a shoulder peak at $345 \mathrm{~nm}$ decreases, which is the same as that observed with pure MINO. The zeta potential of Au NBPs is $+30.1 \mathrm{mV}$; after coating with silica shell, this decreases to $-13.2 \mathrm{mV}$ (Figure 1G). Figure $1 \mathrm{H}$ shows the diameter distribution of Au NBPs and Au NBPs@ $@ \mathrm{SiO}_{2}$ as determined by DLS. The Au NBPs exhibited a mean diameter of $109 \mathrm{~nm}$. After coating silica shell, the diameter increased to $122 \mathrm{~nm}$. Compared with pure nanoparticles, the hybrid nanomaterial integrates various properties into a unique nanoparticle formulation, representing a potential improvement on the previously established therapeutic agent. In this hybrid structure, $\mathrm{Au}$ NBPs exhibit excellent chemical stability, and tunable absorption wavelengths, which could potentially function as a hyperthermia agent for photothermal therapy. Moreover, mesoporous silica has versatile surface chemistry for further functionalization, which can be used as the ideal platform for constructing multifunctional materials.

\section{Characterization of GelMA-Au $\mathrm{NBPs@ \textrm {SiO } _ { 2 }}$}

Although many effective drug release systems have been developed to target bacterial infections, only a few can be 
used for the clinical treatment of periodontitis. Specifically, the development of ideal drug release systems for periodontal pockets remains necessary. ${ }^{22}$ As semi-solid nanomaterials, hydrogels are much easier to prepare and administer than most typical solid nanomaterials, and exhibit good biocompatibility and bioadhesion, ${ }^{23}$ which enables them to easily attach to periodontal pockets. As shown in Figure 2A, the surface of GelMA-AuNBPs@ $\mathrm{SiO}_{2}$ is rough, with varying pore sizes. Additional details of the internal structure are shown in Figure 2B, which indicates a broad pore size distribution from 40 to $72 \mathrm{~nm}$. Compared with the pure GelMA hydrogel, the rheometer of GelMA-Au NBPs@ $\mathrm{SiO}_{2}$ hydrogel reduces to $50 \%$ after adding $\mathrm{Au}$ NBPs@ $\mathrm{SiO}_{2}$ (Figure 2C). By adjusting the concentrations of prepolymer and photoinitiator, elastic modulus and adhesive strength of GelMA hydrogel can be tuned. ${ }^{24}$ The $10 \% \mathrm{w} / \mathrm{v}$ GelMA pre-polymers have excellent adhesion properties, which makes the hydrogel effectively adhere to the surface of the substrate and conducive to long-term existence. As shown in Figure 2D, during NIR light irradiation, the surface temperatures of GelMA-AuNBPs@ $\mathrm{SiO}_{2}$ hydrogel rose rapidly and reached a plateau after irradiation with $1.2 \mathrm{~W} /$ $\mathrm{cm}^{2}$ laser power for 5 mins. Upon 5 mins of NIR laser irradiation $\left(1.2 \mathrm{~W} / \mathrm{cm}^{2}\right)$, the maximal temperature of the hydrogels with different concentration of embedded $\mathrm{Au}$ $\mathrm{NBPs} @ \mathrm{SiO}_{2}$ was $42.1{ }^{\circ} \mathrm{C}, 48.7{ }^{\circ} \mathrm{C}, 52.1{ }^{\circ} \mathrm{C}$, and $57.5{ }^{\circ} \mathrm{C}$, respectively (Figure 2E). Obviously, larger amounts of $\mathrm{Au}$ NBPs@ $\mathrm{SiO}_{2}$ in the hybrid hydrogels lead to a higher temperature during NIR irradiation as a result of higher absorption of NIR by Au NBPs@ $\mathrm{SiO}_{2}$, indicating concentration-dependent features. The hyperthermia shown in Figure $2 \mathrm{~F}$ illustrates that after embedding with $\mathrm{Au} \mathrm{NBPs} @ \mathrm{SiO}_{2}$, NIR light is absorbed and then converted into heat via the photothermal effects of Au NBPs@ $\mathrm{SiO}_{2}$ in the hydrogels. In addition, Au NBPs have unique optical properties compared with other gold nanoparticles (such as gold nanorods, gold nanostars, and gold nanocages). Theoretical calculations show that the photothermal effect of Au NBPs is several times stronger than that of gold nanorods, ${ }^{25,26}$ and the sharpness of the two ends of Au NBPs is directly proportional to the local plasmon resonance effect in the near-infrared range. $^{27}$

\section{Release of Minocycline in vitro}

Figure $3 \mathrm{~A}$ shows the cumulative amounts of MINO released from the hydrogels in PBS buffer $(\mathrm{pH}=7.4)$ at $37^{\circ} \mathrm{C}$. A rapidrelease behavior is observed in the first 2 days, with a relatively slow release in the subsequent 5 days. When the MINO released from the hydrogel in collagenase solution, the release rate is slightly accelerated in comparison with the hydrogels immersed in PBS buffer (Figure 3B). GelMA hydrogels exhibit enzymatic degradation properties. And they retain enzyme-sensitive sequences as the same as both gelatin and collagen. As the hydrogel is immersed in the collagenase solution, the degradation rate of GelMA hydrogels is faster than immersion in PBS buffer. ${ }^{24,28}$ After
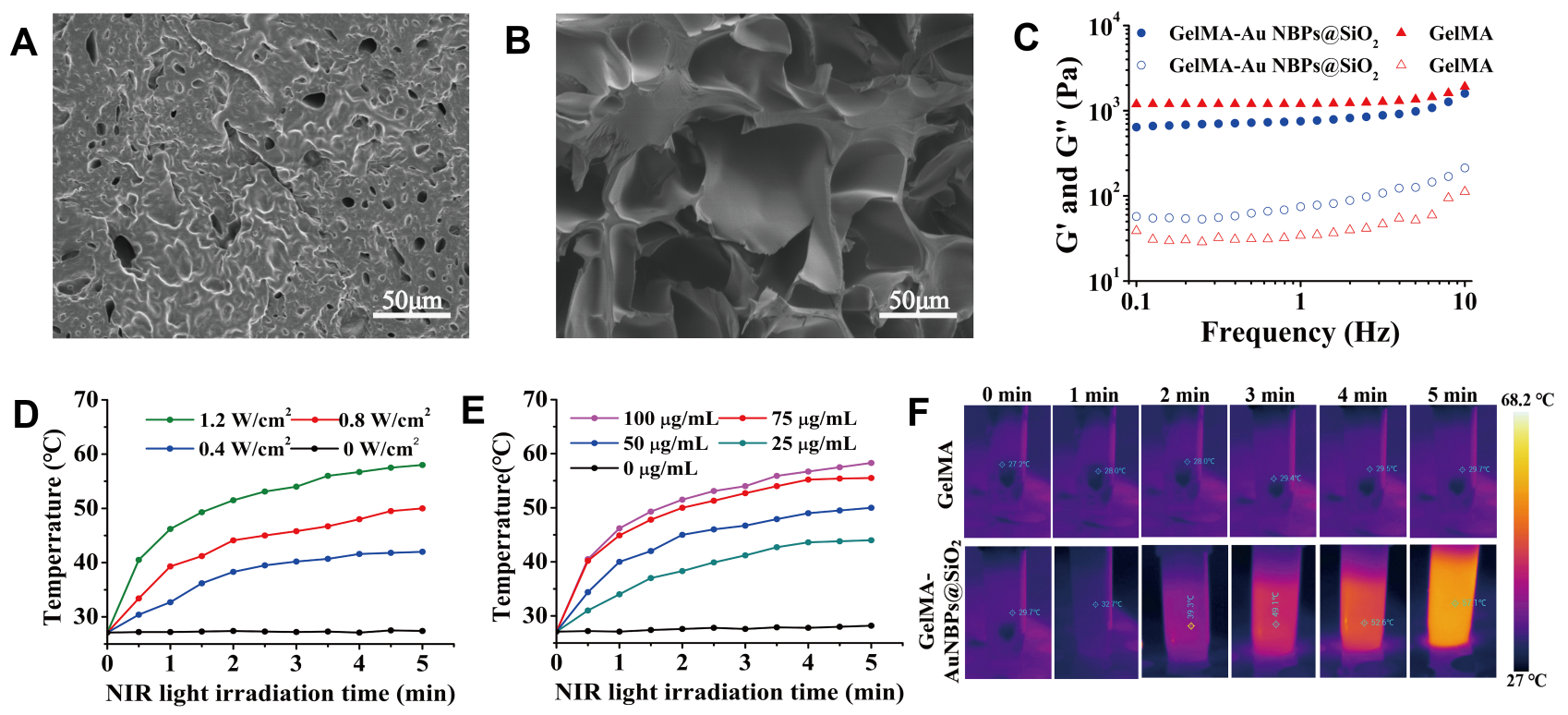

Figure 2 Characterization of GelMA-Au NBPs@SiO 2 . (A) SEM image of surface morphology and (B) the internal structure. Scale bar = $50 \mu$ m. (C) The loss modulus G" and the storage modulus G'. Photothermal properties under $808 \mathrm{~nm}$ laser irradiation at different concentrations (D) and powers (E). Real-time infrared thermal images of GelMA and GelMA-Au NBPs@SiO 2 (F). 

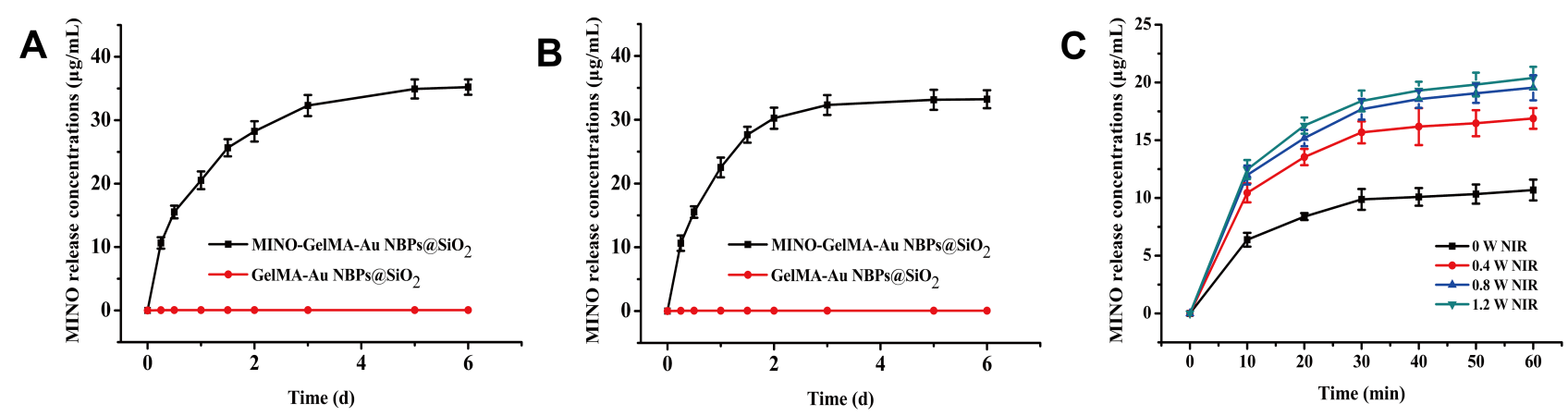

Figure 3 Release curves of minocycline in vitro. (A) Cumulative amounts of MINO released from GelMA-Au NBPs@SiO 2 after immersion in PBS at $37{ }^{\circ} \mathrm{C}$ for 6 days. (B) Cumulative amounts of MINO released from GelMA-Au NBPs@SiO ${ }_{2}$ after immersion in collagenase solution at $37^{\circ} \mathrm{C}$ for 6 days. (C) Cumulative amounts of MINO released from hydrogel with different powers of NIR light. The data is presented as Mean/SD.

activation by NIR light, increased release is observed, which is also dependent on the power of NIR irradiation (Figure 3C): the higher of the power, the greater the molecular release. The maximum release rate was reached when the NIR irradiation power was $1.2 \mathrm{~W} / \mathrm{cm}^{2}$. The NIR-responsive drug release material, which serves as an intelligent drug delivery method, showed controlled drug release in response to stimulation by external factors. The possible reason for this is that as the $\mathrm{Au}$ NBPs@ $\mathrm{SiO}_{2}$-embedded hydrogel absorbs NIR light and converts it into heat, the reduction in volume results in burst release of soluble materials in the hydrogel carrier. It is well known that Au NBPs have prominent NIR-responsiveness. The NIR-responsiveness of $\mathrm{Au} \mathrm{NBPs} @ \mathrm{SiO}_{2}$ nanoparticles can be attributed to the high photothermal conversion efficiency and synergistically enhanced NIR responsiveness derived from Au NBPs. ${ }^{18,29}$ Furthermore, the increased temperature upon laser irradiation is another reason for the high MINO release amount, since the high temperature will accelerate the diffusion and release of drug molecules. ${ }^{30}$ Hence, owing to the temperature response to NIR irradiation, the present hydrogel represents a promising carrier in drug delivery systems.

\section{In vitro Antibacterial Effects}

The NIR light-irradiated samples showed enhanced antibacterial efficacy against $P$. gingivalis. The antibacterial efficacy of the hydrogels rose to $8.7 \%, 51.2 \%$, and $66.7 \%$ against $P$. gingivalis, as the power of NIR light irradiation increased (Figure 4A). In addition, the results in Figure 4B showed the number of viable bacteria were significantly decreased after NIR laser irradiation $\left(1.2 \mathrm{~W} / \mathrm{cm}^{2}\right)$. In comparison, no effect on the bacterial colonies in the control group were observed by Live/Dead staining, regardless of irradiation, indicating that the control group had no antibacterial effect (Figure 4C).
The LIVE/DEAD BacLight Bacterial Viability Kits utilize mixtures of $\mathrm{SYTO}^{\circledR} 9$ green-fluorescent nucleic acid stain and the red-fluorescent nucleic acid stain, propidium iodide. These stains differ both in their spectral characteristics and in their ability to penetrate healthy bacterial cells. With an appropriate mixture of the SYTO 9 and propidium iodide stains, bacteria with intact cell membranes stain fluorescent green, whereas bacteria with damaged membranes stain fluorescent red. ${ }^{31}$ The modification of Au NBPs could improve bactericidal efficacy in PTT and avoid the minimal cytotoxicity to normal tissues. ${ }^{32}$ Exposure to high temperatures (between 57 and $82{ }^{\circ} \mathrm{C}$ for just 30 mins) is used as an effective sterilization strategy against bacteria, e.g. during pasteurization. ${ }^{33}$ Due to the relatively low thermal conductivity of the cell membrane, the heat generated by Au NBPs (a) $\mathrm{SiO}_{2}$ is directed to the surface of $P$. gingivalis to effectively destroy the $P$. gingivalis cell wall. ${ }^{34}$ The advantages of photothermal therapy include the absence of cytotoxic component production, bacterial resistance, external controllability, and broad-spectrum sterilization. ${ }^{35,36}$ Compared with traditional antibiotic treatments, photothermal antibacterial treatment do not elicit bacterial resistance. Secondly, the light source wavelengths used in photothermal antibacterial treatments have deeper tissue penetration than other light-induced treatments. $^{37}$ Finally, by controlling the specific area and intensity of light exposure, the side effects of photothermal antibacterial treatment against normal tissues can be reduced. $^{38-40}$

In comparison, without NIR irradiation, the GelMA-Au NBPs@ $\mathrm{SiO}_{2}$ had no inhibitory effect on bacteria compared with the control group. However, MINO-GelMA-Au

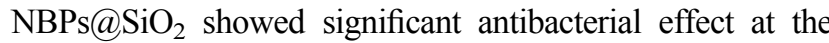
3rd and 5th days, which was as high as $90 \%$ and $67.5 \%$, and maintained for 5 days (Figure 4D). However, the antibacterial 

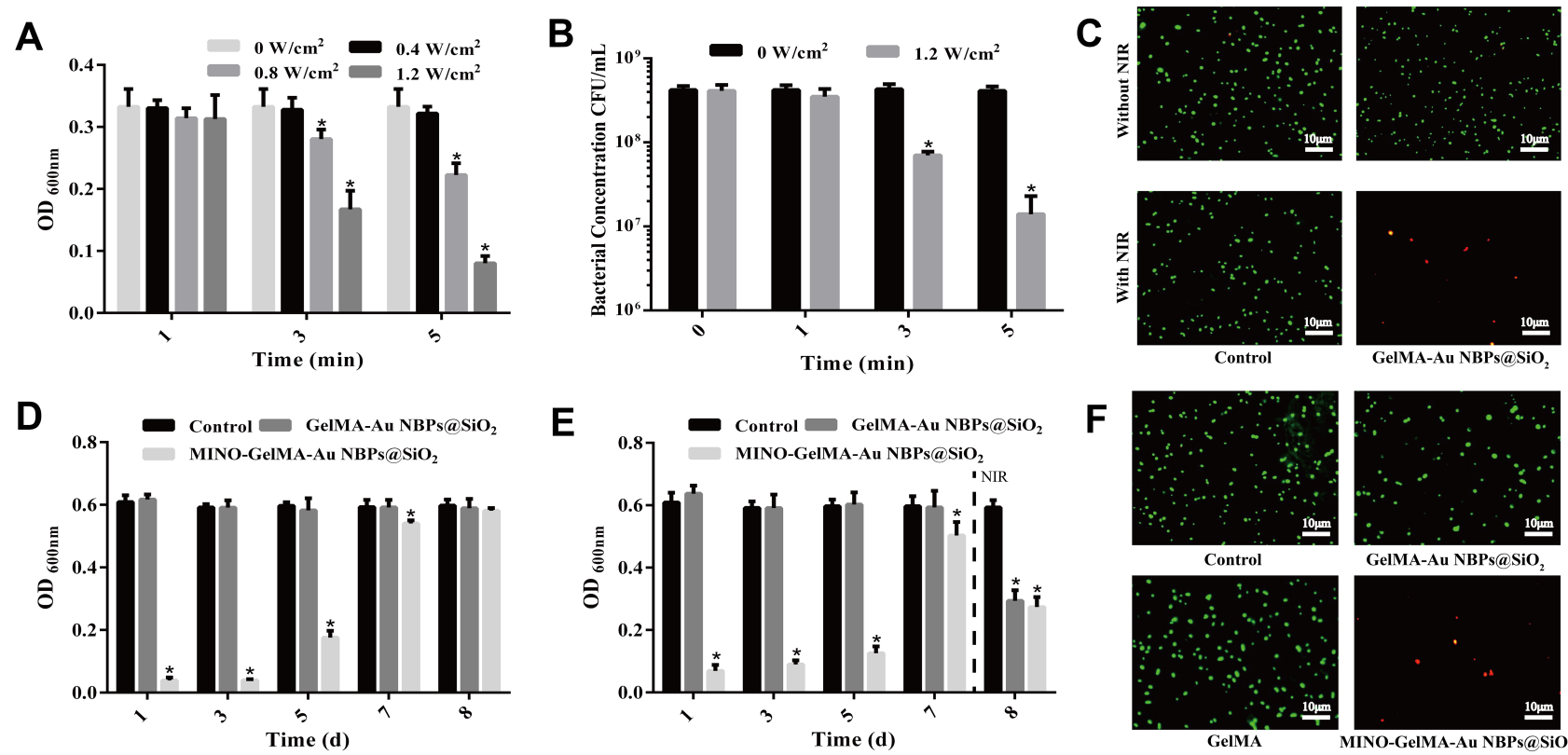

$\mathbf{F}$
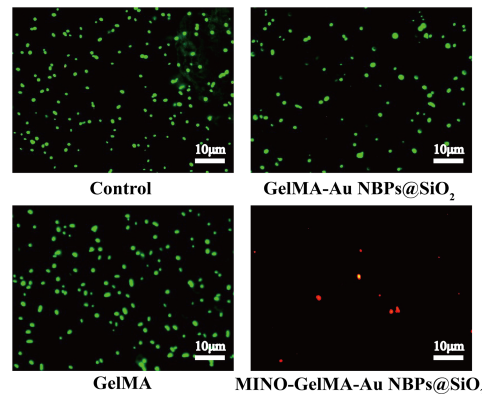

Figure 4 The antibacterial effects of antibiotic and photothermal treatment. (A) The antibacterial effects of photothermal treatment under different power of NIR irradiation. (B) The number of viable bacteria after NIR laser irradiation $\left(I .2 \mathrm{~W} / \mathrm{cm}^{2}\right)$. (C) The live/dead fluorescence images of $P$. gingivalis treated by NIR irradiation. (D) The antibacterial effects of antibiotic at I, 3, 5, 7 and $8 \mathrm{~d}$. (E) The synergistic antibacterial effects of antibiotic and photothermal treatment. (F) The live/dead fluorescence images of $P$. gingivalis treated by different strategies. Scale bar $=10 \mu \mathrm{m}$. The data is presented as Mean/SD: *Compared with control, $\mathrm{p}<0.05$.

efficacy on the 7 th day was only $10 \%$. Subsequently, photothermal treatment improved the antibacterial efficacy to up to $50 \%$ (Figure 4E). Live/Dead staining of bacteria indicated many viable bacteria in the control group and the GelMAAu NBPs@SiO 2 group, while dead bacteria were observed in the MINO-GelMA-Au NBPs@ $\mathrm{SiO}_{2}$ group (Figure 4F). Therefore, the antibacterial effect of MINO-GelMA-Au NBPs@SiO $\mathrm{S}_{2}$ may be supplemented by photothermal antibacterial treatment. After SRP to clear the subgingival pathogens, the residual bacteria present in deep pockets owing to the complex tooth anatomy may not be completely removed, thus reducing the efficacy of periodontal treatment and hindering regeneration of periodontal tissue. We propose the use of MINO-GelMA-Au NBPs@ $\mathrm{SiO}_{2}$ to rapidly clear periodontal pathogens in the periodontal pocket at an early stage with antibiotics, followed by photothermal antibacterial therapy, maintaining low bacterial retention at the maintenance period via physical sterilization. Collectively, the present hydrogels offer a sustained and efficacious antibacterial treatment strategy.

\section{In vitro Cytotoxicity Studies}

For clinical application, biomaterials should possess both antimicrobial capability and biocompatibility. The cytotoxicity of the present hydrogels was investigated by the MTT assay, and biocompatibility was evaluated by co-culture with
L929 cells. As shown in Figure 5A, the hydrogels with different concentrations of embedded Au NBPs@ $\mathrm{SiO}_{2}$ were co-cultured with L929 cells for $24 \mathrm{~h}$ and compared with the control group, which all slightly inhibited the cells. Among them, the $100 \mu \mathrm{g} / \mathrm{mL}$ Au NBPs@ $\mathrm{SiO}_{2}$ group was the most obvious, but the inhibition rate was only $5 \%$. Biocompatibility was further tested using a Live/Dead staining kit. The results are shown in Figure 5A and B: After LIVE/DEAD ${ }^{\mathrm{TM}}$ Viability/Cytotoxicity cell staining, the green fluorescence and red fluorescence indicate live and dead cells, respectively. The control group mainly contains about $99 \%$ viable cells stained green, and about $1 \%$ dead cells are stained red. Each GelMA-Au NBPs@ $\mathrm{SiO}_{2}$ hydrogel group is shown as comprising nearly $98 \%$ viable cells and about $2 \%$ dead cells, indicating a good biocompatibility. GelMA is an attractive photocurable material generated from chemically modified gelatin with methacrylic anhydride (MAA). ${ }^{41}$ In addition, GelMA retains its intrinsic biological properties such as enzymatic degradability and promotion of cell adhesion. ${ }^{42}$ When examining the photothermal antibacterial effect of the hydrogel, it is necessary to consider whether it exerts effects against normal cells under the same conditions. As shown in Figure 5C and D, L929 cells were co-culture with GelMA-Au NBPs@ $\mathrm{SiO}_{2}$ hydrogel and treated by $5 \mathrm{~min}$ of irradiation under $1.2 \mathrm{~W} / \mathrm{cm}^{2}$ power NIR light, and the cell viability was over $80 \%$. It shows that 


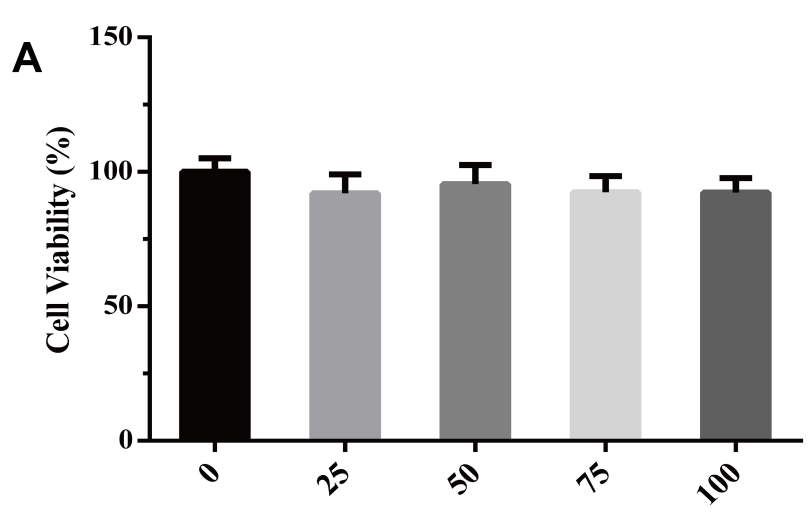

Concentration of Au NBPs@SiO $(\mu \mathrm{g} / \mathrm{mL})$

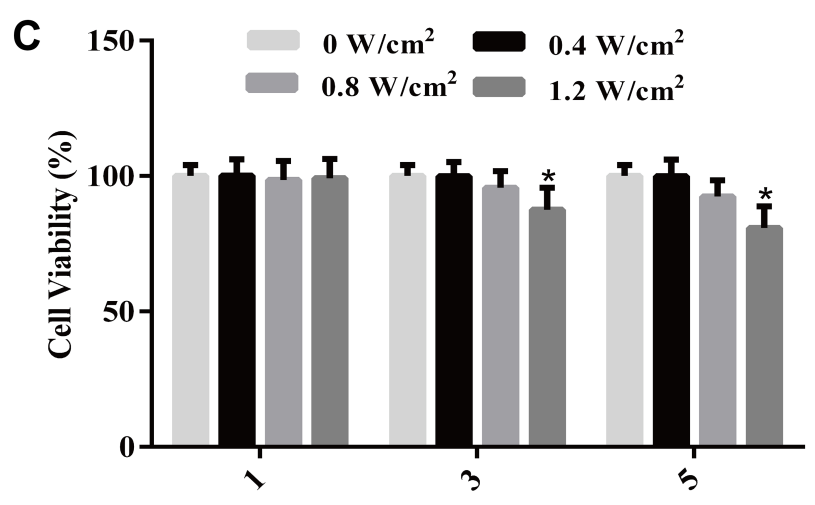

Time (min)
B

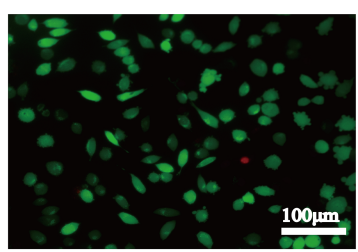

$0 \mu \mathrm{g} / \mathrm{mL} \mathrm{Au}$ NBPs@ $\mathrm{SiO}_{2}$

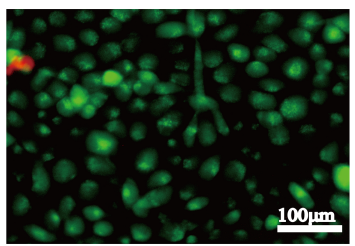

$75 \mu \mathrm{g} / \mathrm{mL}$ Au NBPs@SiO
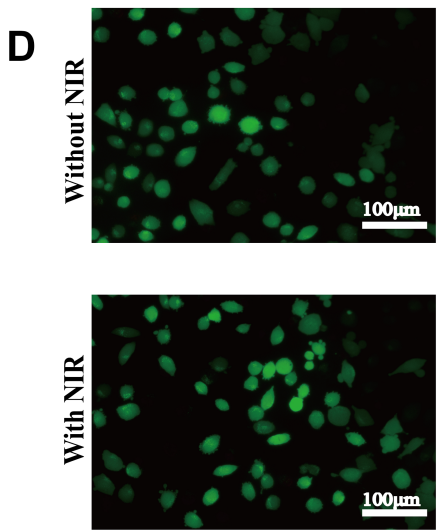

Control

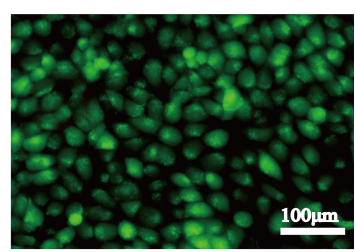

$50 \mu \mathrm{g} / \mathrm{mL} \mathrm{Au}$ NBPs@ $\mathrm{SiO}_{2}$

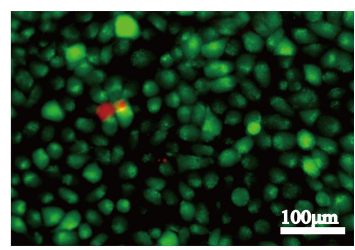

$100 \mu \mathrm{g} / \mathrm{mL}$ Au NBPs@SiO
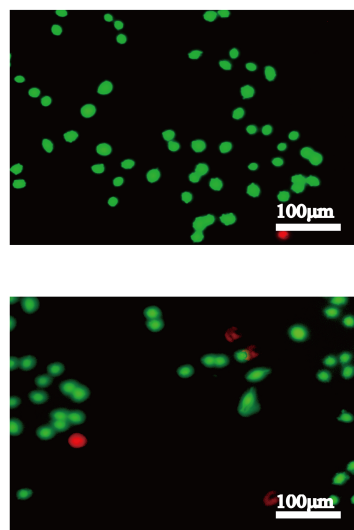

GelMA-Au NBPs@SiO

Figure 5 The biocompatibility of hydrogel and cell viability under NIR irradiation. (A) Cell viability of L929 fibroblast cells after treatment with different concentrations of Au NBPs@SiO 2 . (B) Live/dead fluorescence images of L929 fibroblast cells after treatment with different concentrations of Au NBPs@SiO2. (C) Cell viability of L929 fibroblast cells after treatment with different powers of NIR irradiation. (D) Live/dead fluorescence images of L929 fibroblast cells after treatment with NIR irradiation. Scale bar $=100 \mu \mathrm{m}$. The data is presented as Mean/SD: *Compared with control, $\mathrm{p}<0.05$.

short periods of NIR can affect cell growth, but not completely.

\section{Conclusion}

In summary, we constructed a NIR-activated GelMA-Au NBPs@ $\mathrm{SiO}_{2}$ hybrid hydrogel that enables both antibiotic drug release and physical photothermal treatment under NIR irradiation. The biomaterials possess antibacterial efficacy of $90 \%$ and $66.7 \%$ against $P$. gingivalis by antibiotic and photothermal treatment, respectively. And the rate of MINO release can be controlled by the NIR light. Compared with UV light, NIR has relatively small absorption and scattering coefficients in biological tissues and is therefore preferred in clinical applications. Moreover, the present GelMA-Au NBPs@ $\mathrm{SiO}_{2}$ hydrogels, with synergetic effects due to antibiotics and physical sterilization, show significant promise for the long-term treatment of periodontal disease, with high efficacy.

\section{Acknowledgments}

The research was funded by the scientific research fund of the National Health Commission (wkj-zj-1707); Zhejiang Provincial Science and Technology Project for Public Welfare (LGF20H140003); Zhejiang Provincial Medical and Health Science and Technology Plan (2019KY444); Wenzhou basic scientific research project (2019Y0285); and Natural Science Foundation of Zhejiang Province (Y15H140003).

\section{Disclosure}

The authors report no conflicts of interest in this work.

\section{References}

1. Kinane DF, Stathopoulou PG, Papapanou PN. Periodontal diseases. Nat Rev Dis Primers. 2017;3(1):17038. doi:10.1038/nrdp.2017.38

2. Li S, Schmalz G, Schmidt J, et al. Antimicrobial peptides as a possible interlink between periodontal diseases and its risk factors: a systematic review. J Periodontal Res. 2018;53(2):145-155. doi:10.1111/jre.12482 
3. Oliveira RRDS, Fermiano D, Feres M, et al. Levels of candidate periodontal pathogens in subgingival biofilm. J Dent Res. 2016;95 (6):711-718. doi:10.1177/0022034516634619

4. Warinner C, Rodrigues JFM, Vyas R, et al. Pathogens and host immunity in the ancient human oral cavity. Nat Genet. 2014;46 (4):336-344. doi:10.1038/ng.2906

5. Zupančič Š, Casula L, Rijavec T, et al. Sustained release of antimicrobials from double-layer nanofiber mats for local treatment of periodontal disease, evaluated using a new micro flow-through apparatus. J Control Release. 2019;316:223-235. doi:10.1016/j. jconrel.2019.10.008

6. Keestra JAJ, Grosjean I, Coucke W, et al. Non-surgical periodontal therapy with systemic antibiotics in patients with untreated chronic periodontitis: a systematic review and meta-analysis. $J$ Periodontal Res. 2015;50(3):294-314. doi:10.1111/jre.12221

7. Rajeshwari HR, Dhamecha D, Jagwani S, et al. Local drug delivery systems in the management of periodontitis: a scientific review. J Control Release. 2019;307:393-409. doi:10.1016/j.jconrel.2019.06.038

8. Sarwar H, Khalid HM, Basher MK, et al. Smart nanocarrier-based drug delivery systems for cancer therapy and toxicity studies: a review. $J$ Adv Res. 2019;15:1-18. doi:10.1016/j.jare.2018.06.005

9. Liu J, Luo Z, Zhang J, et al. Hollow mesoporous silica nanoparticles facilitated drug delivery via cascade $\mathrm{pH}$ stimuli in tumor microenvironment for tumor therapy. Biomaterials. 2016;83:51-65. doi:10.1016/j.biomaterials.2016.01.008

10. Shen Y, Li M, Liu T, et al. A dual-functional HER2 aptamer-conjugated, $\mathrm{pH}$-activated mesoporous silica nanocarrier-based drug delivery system provides in vitro synergistic cytotoxicity in HER2-positive breast cancer cells. Int $J$ Nanomedicine. 2019;14:4029-4044. doi:10.2147/IJN. S201688

11. Hernández MA, Llopis-Lorente A, Gorbe $M$, et al. Janus gold nanostars-mesoporous silica nanoparticles for NIR-light-triggered drug delivery. Chemistry. 2019;25:8471-8478. doi:10.1002/ chem. 201900750

12. Huang L, Wu J, Liu M, et al. Direct surface grafting of mesoporous silica nanoparticles with phospholipid choline-containing copolymers through chain transfer free radical polymerization and their controlled drug delivery. $J$ Colloid Interface Sci. 2017;508:396-404. doi:10.1016/j.jcis.2017.08.071

13. Cui J, Sun B, Lin T, et al. Enzyme shielding by mesoporous organosilica shell on Fe3O4@silica yolk-shell nanospheres. Int J Biol Macromol. 2018;117:673-682. doi:10.1016/j.ijbiomac.2018.05.227

14. Lin H, Wang Y, Gao S, et al. Theranostic 2D TANTALUM carbide (MXene). Adv Mater Weinheim. 2018;30(4):1703284. doi:10.1002/ adma.201703284

15. Shao J, Ruan C, Xie H, et al. Black-Phosphorus-Incorporated hydrogel as a sprayable and biodegradable photothermal platform for postsurgical treatment of cancer. Adv Sci. 2018;5(5):1700848. doi:10.1002/advs.201700848

16. Liu Y, Guo Z, Li F, et al. Multifunctional magnetic copper ferrite nanoparticles as fenton-like reaction and near-infrared photothermal agents for synergetic antibacterial therapy. ACS Appl Mater Interfaces. 2019;11(35):31649-31660. doi:10.1021/acsami.9b10096

17. Li C, Mei E, Chen C, et al. Gold-nanobipyramid-based nanotheranostics for dual-modality imaging-guided phototherapy. ACS Appl Mater Interfaces. 2020;12(11):12541-12548. doi:10.1021/ acsami.0c00112

18. Feng J, Chen L, Xia Y, et al. Bioconjugation of gold nanobipyramids for SERS detection and targeted photothermal therapy in breast cancer. ACS Biomater Sci Eng. 2017;3(4):608-618. doi:10.1021/ acsbiomaterials.7b00021

19. Feng J, Wang Z, Shen B, et al. Effects of template removal on both morphology of mesoporous silica-coated gold nanorod and its biomedical application. RSC Adv. 2014;4(54):28683. doi:10.1039/ c4ra03122a
20. Zhou L, Liu Z, Zhang H, et al. Site-specific growth of AgPd nanodendrites on highly purified Au bipyramids with remarkable catalytic performance. Nanoscale. 2014;6(21):12971-12980. doi:10.1039/ c4nr04190a

21. Liu J, Liang H, Li M, et al. Tumor acidity activating multifunctional nanoplatform for NIR-mediated multiple enhanced photodynamic and photothermal tumor therapy. Biomaterials. 2018;157:107-124. doi:10.1016/j.biomaterials.2017.12.003

22. Park J-S, Lim Y-M, Baik J, et al. Preparation and evaluation of $\beta$ glucan hydrogel prepared by the radiation technique for drug carrier applications. Int J Biol Macromol. 2018;118:333-339. doi:10.1016/j. ijbiomac.2018.06.068

23. Zhou J, Yao D, Qian Z, et al. Bacteria-responsive intelligent wound dressing: simultaneous In situ detection and inhibition of bacterial infection for accelerated wound healing. Biomaterials. 2018;161:11-23. doi:10.1016/j.biomaterials.2018.01.024

24. Choi JR, Yong KW, Choi JY, et al. Recent advances in photo-crosslinkable hydrogels for biomedical applications. Biotechniques. 2019;66(1):40-53. doi:10.2144/btn-2018-0083

25. Chateau D, Liotta A, Vadcard F, et al. From gold nanobipyramids to nanojavelins for a precise tuning of the plasmon resonance to the infrared wavelengths: experimental and theoretical aspects. Nanoscale. 2015;7(5):1934-1943. doi:10.1039/c4nr06323f

26. Campu A, Lerouge F, Chateau D, et al. Gold nanobipyramids performing as highly sensitive dual-modal optical immunosensors. Anal Chem. 2018;90(14):8567-8575. doi:10.1021/acs.analchem.8b01689

27. Wang J, Wang S, Mi L, et al. Aspect ratio dependence of the enhancement of fluorescence intensity by gold nanobipyramids for cancer cell imaging and photodynamic therapy. Laser Phys. 2018;28 (7):075602. doi:10.1088/1555-6611/aabdb2

28. Kang Y-F, Zheng B, Li C-Y, et al. Real-time monitoring of temperature variations around a gold nanobipyramid targeted cancer cell under photothermal heating by actively manipulating an optically trapped luminescent upconversion microparticle. Anal Chem. 2020;92(1):1292-1300. doi:10.1021/acs.analchem.9b04470

29. Dolinina ES, Akimsheva EY, Parfenyuk EV. Development of novel silica-based formulation of $\alpha$-lipoic acid: evaluation of photo and thermal stability of the encapsulated drug. Pharmaceutics. 2020;12 (3):228. doi:10.3390/pharmaceutics12030228

30. Zhu M, Wang Y, Ferracci G, et al. Gelatin methacryloyl and its hydrogels with an exceptional degree of controllability and batch-tobatch consistency. Sci Rep. 2019;9(1):6863. doi:10.1038/s41598-01942186-x

31. Boulos L, Prévost M, Barbeau B, et al. LIVE/DEAD ${ }^{\circledR}$ BacLight $^{\mathrm{TM}}$ : application of a new rapid staining method for direct enumeration of viable and total bacteria in drinking water. $J$ Microbiol Methods. 1999;37(1):77-86. doi:10.1016/s0167-7012(99)00048-2

32. Rao W, Li Q, Wang Y, et al. Comparison of photoluminescence quantum yield of single gold nanobipyramids and gold nanorods. ACS Nano. 2015;9(3):2783-2791. doi:10.1021/nn506689b

33. Zhao Q, Wang X, Yan Y, et al. The advantage of hollow mesoporous carbon as a near-infrared absorbing drug carrier in chemo-photothermal therapy compared with IR-820. Eur J Pharm Sci. 2017;99:66-74. doi:10.1016/j.ejps.2016.11.031

34. Teng CP, Zhou T, Ye E, et al. Effective targeted photothermal ablation of multidrug resistant bacteria and their biofilms with nir-absorbing gold nanocrosses. Adv Health Mater. 2016;5 (16):2122-2130. doi:10.1002/adhm.201600346

35. Li M, Liu X, Tan L, et al. Noninvasive rapid bacteria-killing and acceleration of wound healing through photothermal/photodynamic/ copper ion synergistic action of a hybrid hydrogel. Biomater Sci. 2018;6(8):2110-2121. doi:10.1039/c8bm00499d

36. Wu S, Li A, Zhao X, et al. Silica-coated gold-silver nanocages as photothermal antibacterial agents for combined anti-infective therapy. ACS Appl Mater Interfaces. 2019;11(19):17177-17183. doi:10.1021/ acsami.9b01149 
37. Zhu X, Feng W, Chang J, et al. Temperature-feedback upconversion nanocomposite for accurate photothermal therapy at facile temperature. Nat Commun. 2016;7(1):10437. doi:10.1038/ncomms10437

38. Xu J-W, Yao K, Xu Z-K. Nanomaterials with a photothermal effect for antibacterial activities: an overview. Nanoscale. 2019;11 (18):8680-8691. doi:10.1039/c9nr01833f

39. Wang S-G, Chen Y-C, Chen Y-C. Antibacterial gold nanoparticle-based photothermal killing of vancomycin-resistant bacteria. Nanomedicine. 2018;13(12):1405-1416. doi:10.2217/nnm-2017-0380

40. Zhang L, Wang Y, Wang J, et al. Photon-responsive antibacterial nanoplatform for synergistic photothermal-/pharmaco-therapy of skin infection. ACS Appl Mater Interfaces. 2019;11(1):300-310. doi:10.1021/acsami.8b18146
41. Lee B, Lum N, Seow L, et al. Synthesis and characterization of types A and B gelatin methacryloyl for bioink applications. Materials. 2016;9(10):797-810. doi:10.3390/ma9100797

42. Rehman SRU, Augustine R, Zahid AA, et al. Reduced graphene oxide incorporated GelMA hydrogel promotes angiogenesis for wound healing applications. Int J Nanomedicine. 2019;14:9603-9617. doi:10.2147/ IJN.S218120

\section{Publish your work in this journal}

The International Journal of Nanomedicine is an international, peerreviewed journal focusing on the application of nanotechnology in diagnostics, therapeutics, and drug delivery systems throughout the biomedical field. This journal is indexed on PubMed Central, MedLine, CAS, SciSearch ${ }^{\mathbb{R}}$, Current Contents ${ }^{\mathbb{R}} /$ Clinical Medicine,
Journal Citation Reports/Science Edition, EMBase, Scopus and the Elsevier Bibliographic databases. The manuscript management system is completely online and includes a very quick and fair peer-review system, which is all easy to use. Visit http://www.dovepress.com/ testimonials.php to read real quotes from published authors. 